\title{
Human Umbilical Cord Perivascular Cell
}

National Cancer Institute

\section{Source}

National Cancer Institute. Human Umbilical Cord Perivascular Cell. NCI Thesaurus. Code C124146.

Mesenchymal stem cells isolated from umbilical cord blood capable of high proliferation potential and colony formation capacity in vitro and the ability to proliferate and differentiate to an osteogenic phenotype in culture. 